\title{
A non-calculus approach to solving the utility maximization problem using the Cobb-Douglas and CES utility function
}

\author{
Vedran Kojić ${ }^{1, *}$ \\ ${ }^{1}$ Faculty of Economics and Business, University of Zagreb \\ Trg J. F. Kennedyja 6, 10000 Zagreb, Croatia \\ E-mail: 〈vkojic@efzg.hr〉
}

\begin{abstract}
This paper proposes a new original non-calculus method to solving the utility maximization problem using the Cobb-Douglas and the CES utility functions, and incorporating the weighted arithmetic-geometric-mean inequality (weighted AM-GM inequality) and Jensen's inequality. Instead of using calculus, the substitution method or the Lagrange multiplier method, the maximum utility and global maximizer for the case of the Cobb-Douglas and CES utility functions are derived in a direct way. The new method does not require checking first and second order conditions, which the substitution method and the Lagrange multiplier method normally require.
\end{abstract}

Keywords: constrained optimization, Cobb-Douglas utility function, CES utility function, non-calculus approach, mathematical inequalities

Received: September 22, 2014; accepted: March 18, 2015; available online: March 30, 2015

DOI: $10.17535 /$ crorr.2015.0021

\section{Introduction}

Differential calculus is a powerful tool for solving various optimization problems not only in mathematics but in economics as well. However, its implementation is not always simple. Finding stationary points of a given function with several variables by solving a system of equations can pose a significant problem, especially if the system is nonlinear. Furthermore, checking the definiteness of the associated Hessian matrix is not always straightforward. To avoid difficulties in differential calculus, mathematicians have endeavored to find alternative ways and approaches to solving optimization problems. The first of these papers was The Solution of Problems in Maxima and Minima by Algebra by Graver [4] and Maxima and Minima without Calculus by Niven [10]. To solve certain optimization problems without calculus they used an algebraic method, which "requires only knowledge of simple factorization such as completing the perfect trinomial and squared binomial, which are considered elementary

${ }^{*}$ Corresponding author. 
mathematics" (see Cardenás-Barrón [3]). Subsequent to those first papers, numerous papers have appeared in which authors have used an algebraic method to solve various inventory models, such as the economic order quantity (EOQ) model, the economic production quantity (EPQ) model, and other models (a good review of papers that deal with inventory models can be found e.g. in [3]). Another approach to solving certain optimization problems without calculus uses mathematical inequalities. The arithmetic-geometric mean inequality and the Cauchy-Buniakowsky-Schwarz inequality are used to derive various inventory models as well (see e.g. [2, 3, 8, 11]). Further, Liu [7] has used a geometric programming approach to solve the profit maximization problem without calculus.

In this paper, Jensen's inequality and the weighted arithmetic-geometric mean (AM-GM) inequality are used to solve the utility maximization problem based on the Cobb-Douglas and CES utility functions. Instead of using calculus and the Lagrange multiplier method, the maximum utility and global maximizer for the case of the Cobb-Douglas and CES utility functions are derived in a direct way. To the best of my knowledge, this original approach has never been used to solve the utility maximization problem.

The rest of the paper is organized as follows: Section 2 provides a theoretical background, explains the motivation behind this paper and provides a simple example of using the arithmetic-geometric mean inequality; Section 3 presents a new approach to solving the utility maximization problem; Section 4 contains conclusions and a note for further research.

\section{Theoretical background and motivation}

To derive a traditional EOQ model without calculus, Teng [11] used the arithmetic-geometric mean inequality for two real positive numbers, say $a$ and $b$ :

$$
\frac{a+b}{2} \geq \sqrt{a b}
$$

The equality in (1) holds if and only if $a=b$. In the traditional EOQ model without backorders, the total relevant cost per unit time, $T C(Q)$, is as follows

$$
T C(Q)=\frac{A d}{Q}+\frac{h Q}{2},
$$

where $Q$ is the order quantity, $A$ is the ordering cost per order and $h$ is the holding cost per unit and unit of time. By using (1), it is easy to obtain the following

$$
T C(Q)=\frac{A d}{Q}+\frac{h Q}{2} \geq \sqrt{2 A d h} .
$$

When the equality $\frac{A d}{Q}=\frac{h Q}{2}$ holds, the optimal order quantity $Q^{*}$ is obtained as 


$$
Q^{*}=\sqrt{2 A d / h}
$$

Thus, the minimum total relevant cost per unit per time, which follows from $(3)$, is

$$
T C\left(Q^{*}\right)=\sqrt{2 A d h}
$$

Seeing that this was a very easy and straightforward approach to solving an important problem in inventory theory, I was motivated to search for other important economic problems that could be solved in an easier way using mathematical inequalities. I came across an important microeconomic problem, the utility maximization problem, which can be solved without calculus, as will be presented later. For the moment, two important mathematical inequalities that will be used are presented below.

Many books deal with mathematical inequalities (see e.g. [1, 5]). This section presents two important mathematical inequalities.

Theorem 1. (Jensen's inequality) If $f$ is strictly convex in $[a, b]$, then for any $t_{1}, t_{2}, \ldots, t_{n} \in[0,1]$, with $\sum_{i=1}^{n} t_{i}=1$, and for $x_{1}, \ldots ., x_{n} \in[a, b]$, we can deduce that

$$
f\left(t_{1} x_{1}+\cdots+t_{n} x_{n}\right) \leq t_{1} f\left(x_{1}\right)+\cdots+t_{n} f\left(x_{n}\right) \text {. }
$$

The equality in (6) holds if and only if $x_{1}=x_{2}=\ldots=x_{n}$. Proof can be found in, e.g. $[1,5]$.

Note: If $f$ is strictly concave function, then (6) becomes

$$
t_{1} f\left(x_{1}\right)+\cdots+t_{n} f\left(x_{n}\right) \leq f\left(t_{1} x_{1}+\cdots+t_{n} x_{n}\right) .
$$

In particular, for $n=2$, (7) becomes

where $t_{1}+t_{2}=1$.

$$
t_{1} f\left(x_{1}\right)+t_{2} f\left(x_{2}\right) \leq f\left(t_{1} x_{1}+t_{2} x_{2}\right),
$$

Theorem 2. (weighted AM-GM inequality) If $x_{1}, \ldots, x_{n}, t_{1}, \ldots, t_{n}$, are positive numbers and $\sum_{i=1}^{n} t_{i}=1$, then

$$
x_{1}^{t_{1}} \cdots x_{n}^{t_{n}} \leq t_{1} x_{1}+\cdots+t_{n} x_{n}
$$

The equality holds if and only if $x_{1}=x_{2}=\ldots=x_{n}$. In particular, for $n=2,(9)$ becomes 


$$
x_{1}^{t_{1}} x_{2}^{t_{2}} \leq t_{1} x_{1}+t_{2} x_{2}
$$

Proof (see [1:27]): Since $x_{i}^{t_{i}}=e^{t_{i} \log x_{i}}$ and $e^{x}$ is convex, by using Jensen's inequality (6), we can deduce that

$$
\begin{aligned}
x_{1}^{t_{1}} \cdots x_{n}^{t_{n}} & =e^{t_{1} \log x_{1}} \cdots e^{t_{n} \log x_{n}}=e^{t_{1} \log x_{1}+\cdots t_{n} \log x_{n}} \\
& \leq t_{1} e^{\log x_{1}}+\cdots t_{n} e^{\log x_{n}}=t_{1} x_{1}+\cdots t_{n} x_{n} .
\end{aligned}
$$

\section{A new approach to solving the utility maximization problem}

Formally, the consumer utility maximization problem is given as

$$
\begin{aligned}
& \max _{\substack{x_{1}, \ldots, x_{n} \geq 0 \\
\text { s.t. }}} \sum_{i=1}^{n} p_{i} x_{i}=y,
\end{aligned}
$$

where $x_{i}$ represents quantity of commodity $i$ that a consumer buys, $p_{i}$ represents price per unit of commodity $i, y$ is a consumer's fixed money income, and $u$ is a strictly increasing and strictly quasiconcave utility function. Thus, the consumer problem (11)-(12) can be cast equivalently as a problem of maximizing the utility function subject to the budget constraint (see [6:20-21]). There are two common methods for solving the problem (11)-(12), well known in mainstream microeconomic literature (see $[6,9,12])$ : the substitution method and the Lagrange multiplier method. Both of these methods use differential calculus. The method proposed here is substitution-based and instead of using calculus uses mathematical inequalities introduced in Section 3. Furthermore, to solve the problem (11)-(12), the utility function needs to be specified. The two most relevant cases, solved in mainstream microeconomic literature (such as $[6,9$, 12]), deal with the CES utility function and the Cobb-Douglas utility function, but only in the case of two commodities, i.e. $n=2$.

\subsection{The CES utility function}

Let us commence by solving the problem (11)-(12) in case of the CES utility function $u\left(x_{1}, x_{2}\right)=\left(x_{1}^{\rho}+x_{2}^{\rho}\right)^{\frac{1}{\rho}}$, where $0<\rho<1$. Thus, (11)-(12) becomes

$$
\begin{aligned}
& \max _{x_{1}, x_{2} \geq 0} u\left(x_{1}, x_{2}\right)=\left(x_{1}^{\rho}+x_{2}^{\rho}\right)^{\frac{1}{\rho}} \\
& \text { s.t. } p_{1} x_{1}+p_{2} x_{2}=y,
\end{aligned}
$$

as stated in [6:25]. From (14), it follows that 


$$
x_{2}=\frac{y}{p_{2}}-\frac{p_{1}}{p_{2}} x_{1} .
$$

Substituting (15) in (13), we transformed the problem (13)-(14) into an unconstrained maximization problem

$$
\max _{x_{1} \geq 0} u\left(x_{1}, x_{2}\right)=\left[x_{1}^{\rho}+\left(\frac{y}{p_{2}}-\frac{p_{1}}{p_{2}} x_{1}\right)^{\rho}\right]^{\frac{1}{\rho}} .
$$

Let $\gamma_{1}, \gamma_{2}>0, t_{1}=\frac{\gamma_{1}}{\gamma_{1}+\gamma_{2}}, t_{2}=\frac{\gamma_{2}}{\gamma_{1}+\gamma_{2}}, t_{1}+t_{2}=1,0<t_{1}, t_{2}<1$. Further, let us transform function $u$ from (16) in the following way:

$$
\begin{aligned}
u\left(x_{1}, x_{2}\right) & =\left[\frac{\gamma_{1}}{\gamma_{1}} x_{1}^{\rho}+\frac{\gamma_{2}}{\gamma_{2}}\left(\frac{y}{p_{2}}-\frac{p_{1}}{p_{2}} x_{1}\right)^{\rho}\right]^{\frac{1}{\rho}}=\left[\left(\gamma_{1}+\gamma_{2}\right)\left(t_{1} \frac{x_{1}^{\rho}}{\gamma_{1}}+t_{2} \frac{1}{\gamma_{2}}\left(\frac{y}{p_{2}}-\frac{p_{1}}{p_{2}} x_{1}\right)^{\rho}\right)\right]^{\frac{1}{\rho}} \\
& =\left(\gamma_{1}+\gamma_{2}\right)^{\frac{1}{\rho}}\left[t_{1}\left(\frac{x_{1}}{\gamma_{1}^{\frac{1}{\rho}}}\right)^{\rho}+t_{2}\left(\frac{\frac{y}{p_{2}}-\frac{p_{1}}{p_{2}} x_{1}}{\gamma_{2}^{\frac{1}{\rho}}}\right)^{\frac{1}{\rho}}\right]^{\rho}
\end{aligned}
$$

Since function $f^{2}(x)=x^{\rho}, x>0$, where $0<\rho<1$, is strictly concave, by using (8), from (17) it follows that

$$
\begin{gathered}
u\left(x_{1}, x_{2}\right) \leq\left(\gamma_{1}+\gamma_{2}\right)^{\frac{1}{\rho}}\left\{\left[t_{1} \frac{x_{1}}{\gamma_{1}^{\frac{1}{\rho}}}+t_{2} \frac{\frac{y}{p_{2}}-\frac{p_{1}}{p_{2}} x_{1}}{\gamma_{2}^{\frac{1}{\rho}}}\right]^{\rho}\right]^{\frac{1}{\rho}} \\
=\left(\gamma_{1}+\gamma_{2}\right)^{\frac{1}{\rho}}\left[t_{1} \frac{x_{1}}{\gamma_{1}^{\frac{1}{\rho}}-t_{2} \frac{\frac{p_{1}}{p_{1}}}{\gamma_{2}^{\frac{1}{\rho}}}+t_{2} \frac{\frac{p_{2}}{\frac{1}{\rho}}}{\gamma_{2}^{\rho}}}\right] .
\end{gathered}
$$

Now, the goal is to make the term $t_{1} \frac{x_{1}}{\gamma_{1}^{\frac{1}{\rho}}}-t_{2} \frac{\frac{p_{1}}{p_{2}} x_{1}}{\gamma_{2}^{\frac{1}{\rho}}}$ from (18) to equal zero for all $x_{1}$. This is possible if and only if

$$
\frac{t_{1}}{\gamma_{1}^{\frac{1}{\rho}}}=\frac{t_{2} \frac{p_{1}}{p_{2}}}{\gamma_{2}^{\frac{1}{\rho}}} \Leftrightarrow \frac{t_{1}}{t_{2}}=\left(\frac{\gamma_{1}}{\gamma_{2}}\right)^{\frac{1}{\rho}} \frac{p_{1}}{p_{2}} .
$$


Combining $t_{1}+t_{2}=1, \frac{t_{1}}{t_{2}}=\frac{\gamma_{1}}{\gamma_{2}},(19)$, and letting $\gamma_{1}=1$, leads simply to the following results

$$
t_{1}=\frac{p_{1}^{\frac{\rho}{\rho-1}}}{p_{1}^{\frac{\rho}{\rho-1}}+p_{2}^{\frac{\rho}{\rho-1}}}, t_{2}=\frac{p_{2}^{\frac{\rho}{\rho-1}}}{p_{1}^{\frac{\rho}{\rho-1}}+p_{2}^{\frac{\rho}{\rho-1}}}, \gamma_{1}=1, \gamma_{2}=\left(\frac{p_{1}}{p_{2}}\right)^{\frac{\rho}{\rho-1}}
$$

Substituting (20) into (18), the maximum utility can be easily obtained:

$$
u_{\max }=y\left(p_{1}^{\frac{\rho}{\rho-1}}+p_{2}^{\frac{\rho}{\rho-1}}\right)^{\frac{1-\rho}{\rho}} .
$$

The maximum value in (21) is acquired if and only if (see (17)):

$$
\frac{x_{1}}{\gamma_{1}^{\frac{1}{\rho}}}=\frac{\frac{y}{p_{2}}-\frac{p_{1}}{p_{2}} x_{1}}{\gamma_{2}^{\frac{1}{\rho}}} .
$$

From (22), (20) and (15), the global maximizers $x_{1}^{*}, x_{2}^{*}$ can be easily obtained for the problem (13)-(14):

$$
\begin{gathered}
x_{1}^{*}=\frac{y p_{1}^{\frac{1}{\rho-1}}}{p_{1}^{\frac{\rho}{\rho-1}}+p_{2}^{\frac{\rho}{\rho-1}}}, \\
x_{2}^{*}=\frac{y p_{2}^{\frac{1}{\rho-1}}}{p_{1}^{\frac{\rho}{\rho-1}}+p_{2}^{\frac{\rho}{\rho-1}}} .
\end{gathered}
$$

The results (23) and (24) have the same form as in [6:26]. As can be seen, using Jensen's inequality (8) means that the maximum utility (21) and the global maximizer (23) and (24) can be derived directly in the same step, which is not the case with calculus-based methods.

Example 1 To illustrate the method proposed in this section, let us consider the following problem

$$
\begin{array}{ll} 
& \max _{x_{1}, x_{2} \geq 0} u\left(x_{1}, x_{2}\right)=\left(\sqrt{x_{1}}+\sqrt{x_{2}}\right)^{2} \\
\text { s.t. } & x_{1}+2 x_{2}=100 .
\end{array}
$$

In this example, $\rho=0.5, p_{1}=1, p_{2}=2, y=100$. Using (20), (21), (23) and (24) enables us to verify that $t_{1}=2 / 3, t_{2}=1 / 3, \gamma_{1}=1, \gamma_{2}=0.5$, $x_{1}^{*}=200 / 3, x_{2}^{*}=50 / 3, u_{\max }\left(x_{1}^{*}, x_{2}^{*}\right)=150$. 
Cobb-Douglas and CES utility function

\subsection{The Cobb-Douglas utility function}

Let us now solve the problem (11)-(12) for the case of the Cobb-Douglas utility function $u\left(x_{1}, x_{2}\right)=x_{1}^{a} x_{2}^{b}$, where $a, b$ are positive numbers that describe consumer preferences. Thus, (11)-(12) become

$$
\begin{array}{ll} 
& \max _{x_{1}, x_{2} \geq 0} u\left(x_{1}, x_{2}\right)=x_{1}^{a} x_{2}^{b} \\
\text { s.t. } & p_{1} x_{1}+p_{2} x_{2}=y,
\end{array}
$$

as stated in [12:93]. As in the previous section, (15) holds. By substituting (15) with (27), we arrive at an unconstrained maximization problem

$$
\max _{x_{1} \geq 0} u\left(x_{1}, x_{2}\right)=x_{1}^{a}\left(\frac{y}{p_{2}}-\frac{p_{1}}{p_{2}} x_{1}\right)^{b} .
$$

Let us transform (29) in the following way:

$$
\begin{aligned}
u\left(x_{1}, x_{2}\right) & =\frac{1}{p_{2}^{b}}\left(x_{1}^{\frac{a}{a+b}}\right)^{a+b}\left[\left(y-p_{1} x_{1}\right)^{\frac{b}{a+b}}\right]^{a+b} \\
& =\frac{1}{p_{1}^{a} p_{2}^{b}}\left[b^{-\frac{a}{a+b}}\left(b p_{1} x_{1}\right)^{\frac{a}{a+b}} a^{-\frac{b}{a+b}}\left(a y-a p_{1} x_{1}\right)^{\frac{b}{a+b}}\right]^{a+b} \\
& =\frac{a^{-b} b^{-a}}{p_{1}^{a} p_{2}^{b}}\left[\left(b p_{1} x_{1}\right)^{\frac{a}{a+b}}\left(a y-a p_{1} x_{1}\right)^{\frac{b}{a+b}}\right]^{a+b} .
\end{aligned}
$$

Since $\frac{a}{a+b}+\frac{b}{a+b}=1$, and by applying the weighted AM-GM inequality

(10) to the last term in (30), we get

$$
u\left(x_{1}, x_{2}\right) \leq \frac{a^{-b} b^{-a}}{p_{1}^{a} p_{2}^{b}}\left[\frac{a}{a+b} b p_{1} x_{1}+\frac{b}{a+b} a y-\frac{b}{a+b} a p_{1} x_{1}\right]^{a+b}=\frac{a^{a} b^{b}}{(a+b)^{a+b}} \frac{y^{a+b}}{p_{1}^{a} p_{2}^{b}} .
$$

Thus, the maximal utility equals

$$
u_{\max }=\frac{a^{a} b^{b}}{(a+b)^{a+b}} \frac{y^{a+b}}{p_{1}^{a} p_{2}^{b}},
$$

and is achieved if and only if

$$
b p_{1} x_{1}=a y-a p_{1} x_{1} .
$$

From (33), we get optimal quantities of commodities 1 and 2 (i.e. global maximizers), respectively:

$$
x_{1}^{*}=\frac{a y}{p_{1}(a+b)}, x_{2}^{*}=\frac{b y}{p_{2}(a+b)} .
$$

The result (34) has the same form as in [12:94]. 
Example 2 To illustrate the method proposed in this section, let us consider the following problem

$$
\begin{aligned}
& \quad \max _{x_{1}, x_{2} \geq 0} u\left(x_{1}, x_{2}\right)=x_{1}^{\frac{1}{3}} x_{2}^{\frac{2}{3}} \\
& \text { s.t. } x_{1}+2 x_{2}=100 .
\end{aligned}
$$

In this example, $a=1 / 3, b=2 / 3, p_{1}=1, p_{2}=2, y=100$. Using (32) and (34), enables us to verify that $u_{\max }=100, x_{1}^{*}=x_{2}^{*}=100 / 3$.

As is evident, the new presented method, which uses mathematical inequalities, does not use calculus, and furthermore, does not require the nontrivial verification of first and second order conditions, which is otherwise necessary in the substitution method and the Lagrange multiplier method. This characteristic gives an advantage to the method that uses mathematical inequalities over calculus-based methods.

\section{Conclusion}

This paper presents a new original method for solving the utility maximization problem for the case of the CES utility function and Cobb-Douglas utility function. Instead of using differential calculus, problems are solved by applying mathematical inequalities: Jensen's inequality and the weighted AM-GM inequality. Since knowledge of calculus is not required, this methodology allows a wider audience not familiar with differential calculus, such as high school students and others, to approach and analyze this important microeconomic problem. Importantly, the use of mathematical inequalities leads to the maximum utility value and associated maximizer in a direct way and thus verfiying sufficient conditions for unique global optima becomes unnecessary. Solving the utility maximization problems for the case of generalized utility functions with $\mathrm{n}>2$ commodities using mathematical inequalities is open to further research.

\section{References}

[1] Bulajich Manfrino, R., Gomez Ortega, J. A., and Valdez Delgado, R. (2009). Inequalities: A Mathematical Olympiad Approach. Basel: Birkhäuser Verlag.

[2] Cardenás-Barrón, L. E. (2010). An easy method to derive EOQ and EPQ inventory models with backorders. Computer and Mathematics with Applications, 59(2), 948952. doi:10.1016/j.camwa.2009.09.013.

[3] Cardenás-Barrón, L. E. (2011). The derivation of EOQ/EPQ inventory models with two backorders costs using analytic geometry and algebra. Applied Mathematical Modelling, 35(5), 2394-2407. doi:10.1016/j.apm.2010.11.053.

[4] Garver, R. (1935). The solution of problems in maxima and minima by algebra. The American Mathematical Monthly, 42(7), 435-437. doi:10.2307/2301296. 
Cobb-Douglas and CES utility function

[5] Hung, P. K. (2007). Secrets in Inequalities: GIL Publishing House.

[6] Jehle, G. A. and Reny, P. J. (2011). Advanced Microeconomic Theory: FT Prentice Hall.

[7] Liu, S.-T. (2006). A geometric programming approach to profit maximization. Applied Mathematics and Computation, 182(2), 1093-1097. doi:10.1016/j.amc.2006.04.061.

[8] Lukač, Z. and Kojić, V. (2013). Rješavanje problema optimizacije u problemima minimizacije troškova i ekonomične količine nabave pomoću nejednakosti između aritmetičke i geometrijske sredine (Solving optimization problems of cost minimization and economic order quantity by using the inequality between arithmetic and geometric mean). Zbornik Ekonomskog fakulteta u Zagrebu, Sveučilište u Zagrebu (The Proceedings of Zagreb Faculty of Economics and Business), Ekonomski fakultet - Zagreb, 11(1), 81-97.

[9] Mas-Colell, A., Whinston, M. D. and Green, J. R. (1995). Microeconomic Theory. New York: Oxford University Press.

[10] Niven, I. (1981). Maxima and Minima without Calculus. The Dolciani Mathematical Expositions. No. 6: The Mathematical Association of America.

[11] Teng, J.-T. (2009). A simple method to compute economic order quantities. European Journal of Operationl Research, 198, 351-353. doi:10.1016/j.ejor.2008.05.019.

[12] Varian, H. L. (2010). Intermediate Microeconomics: A Modern Approach. New York: W. W. Norton \& Company. 